\title{
Sleep Disordered Breathing-induced Endothelial Dysfunction and Its Association with Fetomaternal Outcomes in Preeclampsia
}

\author{
${ }^{1}$ Gulshan Bano, ${ }^{2}$ Jagdish C Suri, ${ }^{3} \mathrm{HP}$ Anand, ${ }^{4} \mathrm{Jyotsna}$ Suri, ${ }^{5}$ Tulsi Adhikari
}

\begin{abstract}
Introduction: There is a close association between sleep disordered breathing (SDB) and preeclampsia (PE). Endothelial dysfunction (ED) is the common final pathway in the development of hypertension in both the conditions.
\end{abstract}

Materials and methods: Totally, 25 women with new-onset hypertension of pregnancy and 25 age-, body mass index (BMI)-, and gestational age-matched normotensive pregnant women were subjected to polysomnography (PSG) and EndoPAT test. The maternal and fetal outcomes of all the subjects were noted.

Results: The SDB and ED occur more frequently in PE (64 vs $24 \% ; p=0.01$ and 72 vs 28\%; $p=0.002$ respectively) compared with normotensive pregnant women. Mean respiratory distress index (RDI) was higher ( $8.28 \pm 7.9$ vs $4.2 \pm 2.7)$ and mean reactive hyperemic index $(\mathrm{RHI})$ lower (1.46 \pm 0.33 vs $1.88 \pm 7.9$; 0.001) in PE. The mean blood pressure (MBP) in cases tended to correlate positively with RDI and negatively with $\mathrm{RHI}$. There was a significant negative correlation between $\mathrm{RDI}$ and $\mathrm{RHI}(\mathrm{r}=-0.637$; $p=0.001$ ). About $87.5 \%$ of the cases who had SDB also had ED, whereas only $12.5 \%$ of the cases who did not suffer from SDB had an ED ( $p=0.02)$, and this trend was also observed in the controls $(p=0.001)$. It was seen on subgroup analysis that the women with $\mathrm{PE}$, who had both SDB and ED, had poorer maternal and fetal outcomes than those who had neither of the conditions.

Conclusion: The SDB and ED are closely associated with $P E$. The ED occurs more frequently and with greater severity in preeclamptic women who have SDB and is associated with adverse fetomaternal outcomes.

Keywords: Adverse fetal outcomes, Adverse maternal outcomes, Endothelial dysfunction, Preeclampsia, Sleep disordered breathing.

How to cite this article: Bano G, Suri JC, Anand HP, Suri J, Adhikari T. Sleep Disordered Breathing-induced Endothelial Dysfunction and Its Association with Fetomaternal Outcomes in Preeclampsia. Indian J Sleep Med 2017;12(4):53-59.

\footnotetext{
${ }^{1}$ Senior Resident, ${ }^{2-4}$ Professor, ${ }^{5}$ Scientist

1,3,4 Department of Gynecology, Vardhman Mahavir Medical College and Safdarjung Hospital, New Delhi, India

${ }^{2}$ Department of Pulmonary, Critical Care \& Sleep Medicine Vardhman Mahavir Medical College and Safdarjung Hospital New Delhi, India

${ }^{5}$ Indian Council of Medical Statistic, New Delhi, India

Corresponding Author: Jyotsna Suri, Professor, Department of Pulmonary, Critical Care \& Sleep Medicine, Vardhman Mahavir Medical College and Safdarjung Hospital, New Delhi India, e-mail: docjcsuri@gmail.com
}

\section{Source of support: Nil \\ Conflict of interest: None}

\section{INTRODUCTION}

Sleep disordered breathing is a condition that is characterized by repeated episodes of partial or complete upper airway obstruction during sleep, leading to sleep disruption and significant oxyhemoglobin desaturation. Anatomical features like a small pharyngeal airway and central ventilatory disorders are thought to be the chief pathogenetic mechanisms responsible for this condition. ${ }^{1}$ The risk factors include obesity, male sex, increasing age, and pregnancy. ${ }^{2}$

The SDB is associated with several cardiovascular complications including hypertension. ${ }^{3}$ It is postulated that the intermittent spells of hypoxia in SDB can give rise to increased sympathetic activity, inflammation, and oxidative stress ultimately leading to ED and hypertension. ${ }^{4}$

It has been seen that the physiological and anatomical changes during pregnancy make a gravid woman more prone to develop SDB. ${ }^{5,6}$ These changes include an estrogenic-mediated nasal congestion, decrease in the functional residual capacity, and changes in the shape of the diaphragm and thorax due to the pressure effect of the expanding uterus. Some of these changes are even more pronounced in $\mathrm{PE}$, which is supported by the strong association between the two conditions..$^{7-9}$

The PE is a multisystem disorder unique to pregnancy and is defined as the occurrence of hypertension (>140/90) after 20 weeks of gestation along with proteinuria and/or features of organ involvement like deranged liver or kidney functions, thrombocytopenia, pulmonary edema, and new-onset visual or cerebral disturbances. ${ }^{10}$ It is prevalent in about 2 to $8 \%$ of pregnant women and is responsible for significant maternal and fetal morbidity and mortality. ${ }^{11}$

The available literature supports the central role of the placenta in the causation of PE. A long-standing hypothesis has been that PE develops as a consequence of immune maladaptation between the mother and fetus, which leads to impaired arterial invasion by trophoblast cells. This results in poor placental perfusion, with resultant hypoxia and tissue oxidative stress, leading to systemic ED and exaggerated inflammatory response. ${ }^{12}$ 
It is, hence, interesting to note that ED is the final common pathogenetic pathway for both PE and SDB. Moreover, both conditions have been associated with similar long-term cardiovascular and metabolic outcomes, leading to adverse maternal and fetal outcomes. ${ }^{7,13}$ This association is further strengthened by the fact that SDB occurs more frequently and with more severity in women with pregnancy-induced hypertension as compared with normotensive, pregnant women even after controlling for BMI, which is a risk factor for both SDB and PE. ${ }^{8}$

It was hypothesized that the respiratory events in women with SDB may contribute to functional abnormality of blood vessels seen in PE. Further, it was hypothesized that the preeclamptic women with both SDB and ED would have more severe maternal and fetal outcomes. To test these hypotheses, this prospective study was performed on a cohort of 25 preeclamptic women and a similar number of normotensive women.

\section{MATERIALS AND METHODS}

\section{Study Design}

A prospective, cohort study conducted over an 18-month period at the Department of Obstetrics and Gynecology in collaboration with Department of Pulmonary, Critical Care and Sleep Medicine, Vardhman Mahavir Medical College and Safdarjung Hospital, New Delhi, India. The study was approved by the Institutional Ethical Committee.

\section{Participants}

Twenty-five preeclamptic women, between 18 and 35 years of age, with a gestation period of more than 28 weeks were randomly selected from the antenatal outpatient department (OPD) after taking written informed consent. A total of 38 patients met the selection criteria, of which seven patients did not give their consent to be part of the study and six patients had PE with severe features and, hence, had to undergo termination of pregnancy the same day. The clinical and demographic features of these patients were similar to those who consented to be part of the study. Women with chronic hypertension and gestational hypertension and those suffering from asthma, chronic rhinitis, and diabetes were excluded from the study.

The control population consisted of 25 age-, gestation-, and BMI-matched normotensive control gravid women attending the antenatal OPD, who consented to be part of the study.

The PE was diagnosed by presence of new onset hypertension with $\mathrm{BP}>140 / 90 \mathrm{~mm} \mathrm{Hg}$, on two occasions, at least 6 hours apart and proteinuria (more than $300 \mathrm{mg}$ in 24 hour / $1+$ on dipstick) and/or presence of the following severe features: $\mathrm{BP}>160 / 110 \mathrm{~mm} \mathrm{Hg}$; platelet count $<100,000 / \mu \mathrm{L}$; liver enzymes raised more than twice normal; creatinine levels $>1.1 \mathrm{mg} / \mathrm{dL}$; pulmonary edema; or new-onset visual or cerebral disturbances. ${ }^{10}$

\section{Subject Evaluation}

A complete evaluation of the subjects was done by delving into their medical, surgical, obstetrics, and sleep history. The BP was measured in the right arm, sitting position; the procedure was repeated after 6 hours, if the initial value was $>140 / 90 \mathrm{~mm} \mathrm{Hg}$. The patients were treated for pregnancy-induced hypertension as per the institutional protocol.

Daytime sleepiness was measured by using the Epworth Sleepiness Scale (ESS), and the subjects were asked to rate their probability of falling asleep in eight different situations on a score of 0 to 3 . An ESS score of more than 10 was considered to be diagnostic of abnormal sleepiness. ${ }^{14-16}$ The Berlin sleep questionnaire was used for the evaluation of SDB and was filled by one of the authors after directly questioning the subject.

\section{Polysomnography}

All the recruited subjects (both cases and controls) underwent an overnight PSG. The parameters recorded in all the subjects were nasal airflow by thermistor; nasal pressure by pressure cannula; oxygen saturation by pulse oximeter; three-channel electroencephalography (F3M2, C3M2, and O1M2); two-channel electrooculography; anterior tibialis and submental electromyography; thoracic and abdominal efforts by strain gauges; and tracheal sound with microphone attached to the neck. ALICE 5 digital PSG system (Respironics, Inc, Murrysville, PA) was used for recording and storing all the signals. All the PSG records were scored by an experienced sleep medicine specialist.

\section{Definitions of Respiratory Parameters}

- Adrop in the peak thermal sensor excursion by $>90 \%$ of the baseline lasting for $>10$ seconds was defined as apnea. ${ }^{17}$

- The drop of nasal pressure signal excursions by $>30 \%$ of the baseline, which lasted $>10$ seconds and was accompanied by a drop in $3 \%$ or more of oxygen saturation from the preevent baseline or an arousal was defined as hypopnea. ${ }^{17}$

- An abrupt shift of EEG frequency including alpha, theta, and/or frequencies $>16 \mathrm{~Hz}$ that lasted at least 3 seconds, with at least 10 seconds of stable sleep preceding the change was scored as an arousal. ${ }^{17}$

- Arousal index was defined as number of arousals per hour of sleep. 
- Respiratory effort related arousal (RERA) was scored when the series of breaths did not meet criteria for an apnea or hypopnea, and there was increasing respiratory effort or flattening of the nasal pressure waveform for at least 10 seconds, resulting in arousal from sleep.

- The RDI was defined as number of apneas, hypopneas, and RERAs per hour of sleep.

- The SDB was defined as an RDI of 5 or more along with symptoms, such as EDS or an RDI $>15$ with or without associated symptoms. The severity of SDB was defined according to the RDI as mild, 5 to 15/ hour; moderate, 16 to $30 /$ hour; and severe, $>30$ / hour. ${ }^{17}$

- Minimum oxygen saturation was the lowest level oxygen saturation recorded during sleep study.

\section{Evaluation of Endothelial Function}

The endothelial function in the same subjects was evaluated through the EndoPAT device. This is a noninvasive technology that captures a beat-to-beat plethysmographic recording of the finger arterial pulse-wave amplitude (PWA) with pneumatic probes. A finger probe was placed on the index finger of each hand, and the peripheral arterial tone was recorded from both hands during the study period. Endothelial function was assessed using the reactive hyperemia technique, with the subject sitting on a comfortable chair with both hands placed at heart level. The baseline measurements were obtained for 5 minutes, which were recorded in resting state; this was followed by 5 minutes of occlusion of one arm, with cuff inflated on the upper arm to suprasystolic pressure $(50 \mathrm{~mm} \mathrm{Hg}$ above systolic pressure) and then was released to induce reactive (flow-mediated) hyperemia, which was measured for 10 minutes. The other hand remained unoccluded as a reference to correct for potential systemic changes. ${ }^{15}$

The endothelial function was calculated as the ratio between the magnitude of the average postobstructive PWA (1.5-2.5 minutes after release of the arterial occlusion) and average 5 minutes of baseline PWA (preocclusion baseline period), corrected to systemic changes (seen at the nonobstructed arm). The EndoPAT measurements were then analyzed with a computerized, automated algorithm. A value of $\mathrm{RHI} \leq 1.67$ was considered as ED. ${ }^{18,19}$
All the subjects were followed until delivery, and maternal and fetal outcomes were noted in terms of intrauterine growth restriction (IUGR), eclampsia, abruption, and hemolysis, elevated liver enzymes, low platelet count (HELLP) syndrome (maternal), APGAR, neonatal intensive care unit (NICU) admission, low birth weight, prematurity, still birth, and neonatal death (fetal).

\section{Statistical Analysis}

Statistical analysis was done using Chi-squared test for qualitative data. For quantitative normal data, we used unpaired t-test, and for quantitative skewed data, MannWhitney $U$ test was used. Power of study was taken as $80 \%$, alpha error was taken as $5 \%$, and confidence interval was taken as $95 \%$. Statistical significance was defined as $\mathrm{p}<0.05$.

\section{RESULTS}

Both study and control groups were well matched for age, BMI, and period of gestation (POG) as shown in Table 1.

It was observed that the preeclamptic women had significantly higher occurrence of SDB and ED compared with normotensive pregnant women (64 vs $24 \%$; $\mathrm{p}=0.01)$ and (72 vs 28\%; $\mathrm{p}=0.002)$ as seen in Table 2 . The mean RDI of study group was higher compared with the control group $(8.23 \pm 7.9$ vs $4.2 \pm 2.7 ; \mathrm{p}=0.025)$, highlighting the fact that SDB was present with more severity in the women with PE. The average RHI of study group was $1.46 \pm 0.33$ compared with control group $1.88 \pm 0.48(p=0.001)$, indicating the association of ED and PE.

In women with PE, who had SDB, there was ED in $87.5 \%$ of the women; whereas this trend was present even in the normotensive pregnant women, wherein $83.3 \%$ of women were suffering from SDB had ED also, clearly highlighting the strong association of SDB and ED in

Table 1: Demographic characteristics of study and control groups

\begin{tabular}{llll}
\hline Characteristic & Study group & Control group & $p$-value \\
\hline Age (years) & $27.5 \pm 3.7$ & $25.9 \pm 3.3$ & 0.324 \\
BMI $\left(\mathrm{kg} / \mathrm{m}^{2}\right)$ & $25.9 \pm 2.8$ & $25.1 \pm 2.9$ & 0.377 \\
POG at recruitment & $31.84 \pm 2.4$ & $32.04 \pm 2.2$ & 0.499 \\
(weeks) & & & \\
\hline
\end{tabular}

Table 2: The SDB and ED among study and control groups

\begin{tabular}{|c|c|c|c|c|c|c|c|}
\hline & $\begin{array}{l}\text { Study group } \\
(n=25)\end{array}$ & $\begin{array}{l}\text { Control group } \\
(n=25)\end{array}$ & & & $\begin{array}{l}\text { Study group } \\
(n=25)\end{array}$ & $\begin{array}{l}\text { Control group } \\
(n=25)\end{array}$ & \\
\hline & $n(\%)$ & $n(\%)$ & $p$-value & & $n(\%)$ & $n(\%)$ & $p$-value \\
\hline SDB present & $16(64)$ & $06(24)$ & 0.01 & ED present & $18(72)$ & $7(28)$ & 0.002 \\
\hline SDB absent & $9(36)$ & $19(76)$ & & ED absent & $7(28)$ & $18(72)$ & \\
\hline Mean RDI ( $\pm S D)$ & $8.23(7.9)$ & $4.2 \pm 2.7$ & 0.025 & Mean RHI ( $( \pm S D)$ & $1.46 \pm 0.33$ & $1.88 \pm 0.48$ & 0.001 \\
\hline
\end{tabular}

SD: Standard deviation 


\begin{tabular}{lllll}
\hline \multicolumn{4}{c}{ Table 3: Association of SDB and ED in PE } \\
\cline { 1 - 2 } & $\begin{array}{l}\text { Study group } \\
(n=25)\end{array}$ & $\begin{array}{l}\text { Control group } \\
(n=25)\end{array}$ & p-value \\
\cline { 2 - 2 } SDB and ED & $n(\%)$ & & $n(\%)$ & 0.007 \\
\cline { 1 - 2 } SDB+, ED+ & $14(56)$ & & $5(20)$ & \\
SDB+, ED- & $2(08)$ & & $1(04)$ & \\
SDB-, ED+ & $4(16)$ & & $2(08)$ & \\
SDB-, ED- & $5(20)$ & $17(68)$ & \\
\hline
\end{tabular}

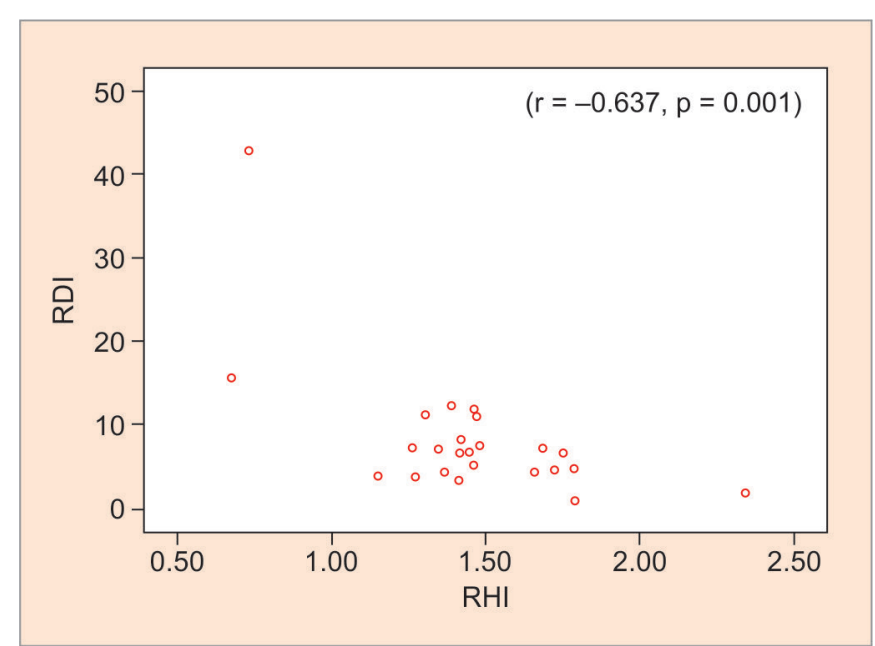

Graph 1: Correlation of RDI (per hour) and RHI in study group

both PE and normotensive pregnant women $(\mathrm{p}=0.021$ and 0.001 respectively).

As many as $56 \%$ of the patients with PE had both SDB and $\mathrm{ED}$, whereas $24 \%$ had either SDB or ED and only $20 \%$ had neither of them, again highlighting the close association of SDB and ED in PE ( $p=0.007)$ (Table 3).

There were positive correlations between MBP and $\operatorname{RDI}(\mathrm{r}=0.205 ; \mathrm{p}=0.326)$ and negative correlation between MBP and RHI ( $r=-0.107 ; p=0.612)$, although it did not reach the level of statistical significance (Table 4). However, this suggested an association of hypertension in preeclampsia with SDB and ED.

The association of RDI and RHI was further strengthened when it was observed that there was a significant negative correlation between the RDI and the RHI in women with PE ( $\mathrm{r}=-0.637, \mathrm{p}=0.001)$ (Graph 1$)$.

Both SDB and ED were found in $56 \%$ of cases compared with only $20 \%$ of controls. A subgroup analysis of preeclamptic patients was done to study the impact of SDB and ED in the causation of adverse maternal and fetal outcomes. The preeclamptic women were divided into two groups, one with both SDB and ED and one without both the conditions, and the fetomaternal outcomes were compared between them.

Preterm birth (78.5 vs 20\%; $\mathrm{p}=0.020)$ and APGAR score $<7$ (71.4 vs $20 \%$; $\mathrm{p}=0.057)$ were higher in preeclamptic women with both SDB and ED compared with
Table 4: Correlation of MBP with RDI and RHI

\begin{tabular}{|c|c|c|c|}
\hline \multirow[b]{2}{*}{ MBP } & \multirow[b]{2}{*}{ Correlation } & \multirow{2}{*}{$\begin{array}{l}R D I(S D B) \\
n=25\end{array}$} & \multirow{2}{*}{$\begin{array}{l}R H I \text { (ED) } \\
n=25\end{array}$} \\
\hline & & & \\
\hline & Pearson correlation & 0.205 & -0.107 \\
\hline & p-value & 0.326 & 0.612 \\
\hline
\end{tabular}

Table 5: Comparison of fetal and maternal outcomes in preeclamptic women with both SDB and ED and preeclamptic women without SDB and ED

\begin{tabular}{llll}
\hline & $\begin{array}{l}\text { Preeclamptic } \\
\text { women with } \\
\text { SDB and ED } \\
(n=14)\end{array}$ & $\begin{array}{l}\text { Preeclamptic } \\
\text { women without } \\
\text { SDB and ED } \\
(n=5)\end{array}$ & p-value \\
\hline $\begin{array}{l}\text { Fetal outcome } \\
\text { IUGR }\end{array}$ & $9(64.3 \%)$ & $1(20 \%)$ & 0.06 \\
Prematurity & $11(78.5 \%)$ & $1(20 \%)$ & 0.020 \\
Low birth weight & $11(78.5 \%)$ & $2(40 \%)$ & 0.17 \\
APGAR score $(<7)$ & $10(71.42 \%)$ & $1(20 \%)$ & 0.057 \\
NICU admission & $9(64.3 \%)$ & $1(20 \%)$ & 0.06 \\
Maternal outcome & & & \\
Abruption & $1(07.14 \%)$ & $0(0 \%)$ & 0.44 \\
Eclampsia & $1(07.14 \%)$ & $0(0 \%)$ & 0.44 \\
HELLP syndrome & $1(07.14 \%)$ & $0(0 \%)$ & 0.44 \\
\hline
\end{tabular}

preeclamptic women without both SDB and ED. Occurrence of IUGR ( 64.3 vs $20 \%$; $\mathrm{p}=0.06)$, low birth weight (78.5 vs $40 \%$; $\mathrm{p}=0.17$ ), and NICU admission ( 64.3 vs $20 \%$; $\mathrm{p}=0.06)$ was also higher in preeclamptic females with SDB and ED, though not statistically significant (Table 5).

When comparing the maternal outcomes between the two subgroups, it was seen that all the adverse outcomes, namely eclampsia, abruption, and HELLP syndrome, occurred more often in preeclamptic women with both SDB and ED compared with preeclamptic women without both SDB and ED (7.14 vs $0.0 \% ; \mathrm{p}=0.44$ for all three outcomes) (Table 5). However, because of the small sample size, statistical significance was not achieved.

\section{DISCUSSION}

The current data suggest higher occurrences of SDB (64 vs $24 \%$; $\mathrm{p}=0.01$ ) and ED (72 vs $28 \%$; $\mathrm{p}=0.002$ ) in preeclampsia compared with normotensive pregnant women. Mean RDI $(8.28 \pm 7.9$ vs $4.2 \pm 2.7)$ was higher and mean RHI lower $(1.46 \pm 0.33$ vs $1.88 \pm 7.9 ; 0.001)$ in PE suggesting the association of SDB and ED with PE. The MBP in cases tended to correlate positively with RDI and negatively with RHI; however, statistical significance was not reached probably due to the fact that 16 of the 25 patients with $\mathrm{PE}$ were on antihypertensives during recruitment. To determine the association of SDB and $\mathrm{ED}$, we analyzed the correlation of RDI and RHI in the cases, and not surprisingly found a significant negative correlation between them $(r=-0.637 ; p=0.001)$, establishing the significant role of SDB and ED in the causation of 
Table 6: Distribution of SDB-induced ED among study and control groups

\begin{tabular}{|c|c|c|c|c|}
\hline \multirow{3}{*}{$\begin{array}{l}\text { Endothelial } \\
\text { function }\end{array}$} & \multicolumn{2}{|c|}{ Study group $(n=25)$} & \multicolumn{2}{|c|}{ Control group $(n=25)$} \\
\hline & $S D B+n t$ & $S D B-n t$ & $S D B+n t$ & $S D B-n t$ \\
\hline & $n(\%)$ & $n(\%)$ & $n(\%)$ & $n(\%)$ \\
\hline Normal & $2(12.5)$ & $5(55.64)$ & $1(16.6)$ & $17(89.5)$ \\
\hline Abnormal & $14(87.5)$ & $4(44.4)$ & $5(83.3)$ & $2(10.5)$ \\
\hline$p$-value & \multicolumn{2}{|c|}{0.021} & \multicolumn{2}{|c|}{0.001} \\
\hline
\end{tabular}

PE. Further, we found that $87.5 \%$ of the cases who had SDB also had ED, whereas only $12.5 \%$ of the cases who did not suffer from SDB had an ED $(p=0.02)$. This trend was also observed in the controls $(p=0.001)$ (Table 6), highlighting the impact of the respiratory events in SDB in inducing ED in all pregnant patients.

As discussed earlier, SDB is seen more commonly and with much more severity in cases of PE. ${ }^{7-9}$ A number of factors may be responsible for the development of SDB in PE. First, the physiological and anatomical changes during pregnancy, which make a gravid woman more prone to SDB, are more exaggerated in PE due to alterations in the upper airway patency, which may be related to the fluid shifts from the lower limbs to the neck area. ${ }^{20-22}$ Increased third space fluid and lower limb edema, which is seen in PE, can result in remarkable fluid shifts from the lower limbs to the neck area in the supine position, leading to more severe upper airway narrowing rendering the upper airway susceptible to collapse during sleep. . $3,24^{2}$ Secondly, obesity, which is a known risk factor for PE, is also an important predisposing factor for SDB. It has been shown that BMI and central fat patterning may independently contribute to SDB. ${ }^{22,25}$ In this study too, we found the mean BMI of PE women to be $25.9 \pm 2.8$.

The respiratory events in SDB are known to result in intermittent hypoxia and increased sympathetic discharge during sleep and wakeful state along with oxidative stress and ED. ${ }^{26}$ All these are postulated to be the critical mediators for cardiovascular disorders in SDB including hypertension. ${ }^{27}$

Preeclampsia is a multisystem disease of pregnancy, with hypertension being its hallmark. ${ }^{10}$ It is postulated that poor placentation resulting in hypoxia, tissue oxidative stress, and ED may be the chief mechanisms for the generalized vasoconstriction and multiorgan involvement in PE, and may also be responsible for the increments in BP. ${ }^{12}$ The results of this study strongly suggest that SDB may be another potentiating factor to induce ED in PE.

It can, hence, be postulated that preeclamptic women who have SDB will have more severe disease, which may be mediated by the ED induced by SDB, and this is reflected by the maternal and fetal outcomes in this study. It was seen on subgroup analysis that the women with PE who had both SDB and ED had poorer maternal and fetal outcomes than those who had neither of the conditions. Prematurity and low APGAR score were significantly higher in the subgroup having SDB and ED. Besides, low birth weight, growth restriction, and NICU admissions were also more often seen in this subgroup having SDB and ED (Table 5). The maternal outcomes like abruption, HELLP, and eclampsia were present in 1 out of 14 patients with SDB and ED as compared with none in those without SDB and ED. The fact that all these PE patients were booked at a tertiary level multidisciplinary hospital where they were instituted protocolized care could be the reason for low rate of adverse maternal outcomes. The other possibility is that fetal outcomes are more vulnerable to the hypoxic events in SDB as compared with maternal outcomes. This trend was also noted by the authors in another study. ${ }^{8}$ Moreover, a larger population needs to be studied to get more definite results in this area.

Similar findings were also demonstrated by Yinon et $\mathrm{al}_{1}^{18}$ wherein they studied 17 women with PE, and concluded that SDB and ED are more likely to occur in them as compared with normotensive controls. However, the major limitation of their study was the small sample size and the fact that SDB was documented by the ambulatory Watch_PAT 100, which is not considered a gold standard for this. Moreover, they did not study any maternal and fetal outcomes except the birth weight of the infant, which they found was significantly lower in the preeclamptic group and also correlated with RDI and RHI.

The strength of this study lies in the fact that all the preeclamptic women and controls underwent a whole night, in-lab PSG, which is considered the gold standard for diagnosis of SDB. Further, the latest noninvasive technology, EndoPAT 2000, was used for determining endothelial function. This has been shown to be a simple, reliable, nonoperator dependent and reproducible tool to assess endothelial vasodilator function..$^{19}$ Being a longitudinal study, the maternal and fetal outcomes could also be assessed.

The main limitation was the small sample size. This was mainly because performing full-night PSG and EndoPAT test in advanced pregnancy had some logistic issues. Moreover, many of the patients with PE had to urgently undergo induction of labor and, hence, could not be recruited in the study.

The clinical significance of this study is that it indicates a potentially treatable factor in women with $\mathrm{PE}$, which is responsible for significant maternal and fetal morbidity and mortality. Delivery of the fetus and placenta have always been established as the definitive management of PE. However, if the PE is associated with SDB, treating that could decrease the severity of ED in PE and also improve maternal and fetal outcomes. There is preliminary 
evidence that administration of CPAP leads to better control of $\mathrm{BP}$ in PE and is also associated with improved fetal outcomes. ${ }^{28,29}$

There is very scant data on the role of SDB and ED in PE. More such studies including interventional studies are urgently warranted, so that the screening and treatment of SDB in PE can become a standard of care in the coming years.

To summarize the findings of this study, it was seen that SDB and ED occurred more frequently and with more severity in $\mathrm{PE}$ as compared with normotensive pregnant women. The RDI and RHI also significantly correlated with each other in the women with PE indicating a close association of PE, SDB, and ED. The ED was seen more commonly and with greater severity in women with PE, who had SDB as compared with those who did not have SDB, suggesting a role of SDB in inducing ED in preeclamptic women though a causal relationship could not be established in this study. Further, the preeclamptic women with SDB and ED had more severe maternal and fetal outcomes as compared with those who did not have either of the two conditions, indicating the contribution of SDB and ED in the poor pregnancy outcomes.

\section{REFERENCES}

1. Rodrigues AP, Pinto P, Nunes B, Bárbara C. Obstructive sleep apnea: epidemiology and Portuguese patients profile. Rev Port Pneumol (2006) 2017 Mar-Apr;23(2):57-61.

2. Mirrakhimov AE, Sooronbaev T, Mirrakhimov EM Prevalence of obstructive sleep apnea in Asian adults: a systematic review of the literature. BMC Pulm Med 2013 Feb;13:10.

3. Gami AS, Olson EJ, Shen WK, Wright RS, Ballman KV, Hodge DO, Herges RM, Howard DE, Somers VK. Obstructive sleep apnea and the risk of sudden cardiac death: a longitudinal study of 10,701 adults. J Am Coll Cardiol 2013 Aug;62(7): 610-616.

4. Namtvedt SK, Hisdal J, Randby A, Agewall S, Stranden E, Somers VK, Røsjø H, Omland T. Impaired endothelial function in persons with obstructive sleep apnoea: impact of obesity. Heart 2013 Jan;99(1):30-34.

5. Izci B, Vennelle M, Liston WA, Dundas KC, Calder AA, Douglas NJ. Sleep disordered breathing and upper airway size in pregnancy and post-partum. Eur Respir J 2006 Feb;27(2):321-327.

6. Suri JC, Sen MK, Suri J, Vaidya S, Adhikari T. Epidemiology of sleep disorders in pregnant patients: a questionnaire based survey. Indian J Sleep Med 2009 Jan;4(3):106-113.

7. Facco FL, Lappen J, Lim C, Zee PC, Grobman WA. Preeclampsia and sleep-disordered breathing: a case-control study. Pregnancy Hypertens 2013 Apr;3(2):133-139.

8. Suri J, Suri JC, Arora R, Gupta M, Adhikari T, The impact of sleep-disordered breathing on severity of pregnancy-induced hypertension and feto-maternal outcomes. J Obstet Gynaecol India 2018 May;1-11.

9. Cain MA, Louis JM. Sleep disordered breathing and adverse pregnancy outcomes. Clin Lab Med 2016 Jun;36(2):435-446.
10. American College of Obstetricians and Gynecologists; Task Force on Hypertension in Pregnancy. Hypertension in pregnancy. Report of the American College of Obstetricians and Gynaecologists' task force on hypertension in pregnancy. Obstet Gynaecol 2013 Nov;122(5):1122-1131.

11. Duley L. The global impact of pre-eclampsia and eclampsia. Semin Perinatol 2009 Jun;33(3):130-137.

12. Mannaerts D, Faes E, Goovaerts I, Stoop T, Cornette J, Gyselaers W, Spaanderman M, Van Craenenbroeck EM, Jacquemyn Y. Flow-mediated dilation and peripheral arterial tonometry are disturbed in preeclampsia and reflect different aspects of endothelial function. Am J Physiol Regul Integr Comp Physiol 2017 Nov;313(5):R518-R525.

13. Louis JM, Mogos MF, Salemi JL, Redline S, Salihu HM. Obstructive sleep apnea and severe maternal-infant morbidity/mortality in the United States, 1998-2009. Sleep 2014 May;37(5):843-849.

14. John MW. A new method for measuring daytime sleepiness: the Epworth Sleepiness Scale. Sleep 1991 Dec;14(6): 540-545.

15. Patel S, Kon SS, Nolan CM, Barker RE, Simonds AK, Morrell MJ, Man WD. The Epworth Sleepiness Scale: minimum clinically important difference in obstructive sleep apnea. Am J Respir Crit Care Med 2018 Apr;197(7):961-963.

16. Li Y, Zhang J, Lei F, Liu H, Li Z, Tang X. Self-evaluated and close relative-evaluated Epworth Sleepiness Scale vs. multiple sleep latency test in patients with obstructive sleep apnea. J Clin Sleep Med 2014 Feb;10(2):171-176.

17. Berry RB, Brooks R, Gamaldo C, Harding SM, Lloyd RM, Quan SF, Troester MT, Vaughn BV. AASM scoring manual updates for 2017 (Version 2.4). J Clin Sleep Med 2017 May;13(5): 665-666.

18. Yinon D, Lowenstein L, Suraya S, Beloosesky R, Zmora O, Malhotra A, Pillar G. Preeclampsia is associated with sleep disordered breathing and endothelial dysfunction. Eur Respir J 2006 Feb;27(2):328-333.

19. Axtell AL, Gomari FA, Cooke JP. Assessing endothelial vasodilator function with the endo-PAT 2000. J Vis Exp 2010 Oct; $44: 2167$.

20. Eiamcharoenwit J, Itthisompaiboon N, Limpawattana P, Suwanpratheep A, Siriussawakul A. The performance of neck circumference and other airway assessment tests for the prediction of difficult intubation in obese parturients undergoing cesarean delivery. Int J Obstet Anesth 2017 May;31: 45-50.

21. Pien GW, Pack AI, Jackson N, Maislin G, Macones GA, Schwab RJ. Risk factors for sleep-disordered breathing in pregnancy. Thorax 2014 Apr;69(4):371-377.

22. Glicksman A, Hadjiyannakis S, Barrowman N, Walker S, Hoey L, Katz SL. Body fat distribution ratios and obstructive sleep apnea severity in youth with obesity. J Clin Sleep Med 2017 Apr;13(4):545-550.

23. Lyons OD, Bradley TD. Heart failure and sleep apnea. Can J Cardiol 2015 Jul;31(7):898-908.

24. Mendelson M, Lyons OD, Yadollahi A, Inami T, OhP, Bradley TD. Effect of exercise training on sleep apnoea in patients with coronary artery disease: a randomized trial. Eur Respir J 2016 Jul;48(1):142-150.

25. Bozkurt NC, Beysel S, Karbek B, Unsal O, Cakir E, Delibasi T. Visceral obesity mediates the association between metabolic syndrome and obstructive sleep apnea syndrome. Metab Syndr Relat Disord 2016 May;14(4):217-221. 
26. Ziegler MG, Milic M, Lu X, Gharaibeh M, Elayan H. Effect of obstructive sleep apnea on the response to hypertension therapy. Clin Exp Hypertens 2017 May;39(5):409-415.

27. Javaheri S, Barbe F, Campos-Rodriguez F, Dempsey JA, Khayat R, Javaheri S, Malhotra A, Martinez-Garcia MA, Mehra R, Pack AI, et al. Sleep apnea: types, mechanisms, and clinical cardiovascular consequences. J Am Coll Cardiol 2017 Feb;69(7):841-858.
28. Blyton DM, Sullivan CE, Edwards N. Reduced nocturnal cardiac output associated with preeclampsia is minimized with the use of nocturnal nasal CPAP. Sleep 2004 Feb;27(1): 79-84.

29. Blyton DM, Skilton MR, Edwards N, Hennessy A, Celermajer DS, Sullivan CE. Treatment of sleep disordered breathing reverses low fetal activity in preeclampsia. Sleep 2013 Jan;36(1): $15-21$. 